\title{
Analgesic Effect of Perioperative Systemic Dexamethasone on Blowout Fracture Surgery
}

\section{Kormi, Eeva}

2017-06

Kormi , E , Snall , J , Koivusalo , A-M , Suominen , A L , Thoren , H \& Tornwall , J 2017 , ' Analgesic Effect of Perioperative Systemic Dexamethasone on Blowout Fracture Surgery ' , Journal of Oral and Maxillofacial Surgery, vol. 75 , no. 6 , pp. 1232-1237 . https://doi.org/10.1016/j.joms.2016.09.02

http://hdl.handle.net/10138/297806

https://doi.org/10.1016/j.joms.2016.09.026

publishedVersion

Downloaded from Helda, University of Helsinki institutional repository.

This is an electronic reprint of the original article.

This reprint may differ from the original in pagination and typographic detail.

Please cite the original version. 


\title{
Analgesic Effect of Perioperative Systemic Dexamethasone on Blowout Fracture Surgery
}

\author{
Eeva Kormi, MD, DDS, *Johanna Snäll, MD, DDS, PhD, † \\ Anna-Maria Koivusalo, MD, PhD, $\ddagger$ Anna Liisa Suominen, DDS, PhD, $\S$ \\ Hanna Thorén, MD, DDS, PbD, // and Jyrki Törnwall, MD, DDS, PhD đ
}

Purpose: To clarify the effect of systemic dexamethasone (DXM) on pain and postoperative opioid (oxycodone) consumption after blowout fracture surgery.

Materials and Methods: A prospective randomized observer-blinded trial of 20 patients who had a blowout fracture requiring surgical intervention was conducted. Patients were randomly assigned to receive a total dose of intravenous DXM $30 \mathrm{mg}$ perioperatively or no DXM (controls). Pain was assessed postoperatively using a 10-cm visual analog scale (VAS) each time analgesics (acetaminophen every 6 hours or oxycodone upon request) were administered. The VAS area under the curve (VAS AUC) for 24 hours postoperatively represented the outcome. Data were analyzed using $\chi^{2}$ test, Student $t$ test, 2-tailed Mann-Whitney $U$ test, and linear regression, with a $P$ value less than .05 indicating significance.

Results: Patients with blowout fracture receiving perioperative systemic DXM exhibited a significantly lower average VAS AUC $(P=.04)$. After controlling for other confounding variables, this result remained significant $(P=.03)$.

Conclusions: DXM appears to decrease postoperative pain and thus is recommended as a pre-emptive analgesic in blowout fracture surgery.

(C) 2017 Published by Elsevier Inc on behalf of the American Association of Oral and Maxillofacial Surgeons

J Oral Maxillofac Surg 75:1232-1237, 2017

Glucocorticoids (GCs) are used to control excessive inflammation because of their established influence on swelling, nausea, and pain. ${ }^{1}$ In analgesia, the action mechanism of GCs could be the inhibition of arachidonic acid metabolism by the suppression of phospholipase $A_{2}$ production and thus the suppression of a wide range of inflammatory mediators. Prostaglandin
$\mathrm{E}_{2}$ (PGE2) is probably the most important mediator for analgesia. ${ }^{2}$ To assess the effect of GCs, many studies have relied on third molar extraction as a clinical model. A meta-analysis on third molar surgical studies showed only a minor positive effect on pain in the early and late postoperative periods. ${ }^{3}$ However, the use of a corticosteroid in oral and maxillofacial surgery
*Resident, Department of Oral and Maxillofacial Diseases, University of Helsinki and Helsinki University Hospital, Helsinki, Finland.

$\dagger$ Resident, Department of Oral and Maxillofacial Diseases, University of Helsinki and Helsinki University Hospital, Helsinki, Finland.

$\ddagger$ Head of Department, Department of Anesthesiology and Intensive Care, University of Helsinki and Helsinki University Hospital, Helsinki, Finland

$\S$ Professor of Oral Health Care and Head of Institute of Dentistry, Institute of Dentistry, University of Eastern Finland, Kuopio, Finland.

$\|$ Consultant and Senior Lecturer, Department of Oral and Maxillofacial Diseases, University of Helsinki and Helsinki University Hospital, Helsinki, Finland.
『Head of Department, Department of Oral and Maxillofacial Diseases, University of Helsinki and Helsinki University Hospital, Helsinki, Finland.

Address correspondence and reprint requests to Dr Kormi: Department of Oral and Maxillofacial Diseases, Helsinki University Hospital, PO Box 220, 00029 HUS, Helsinki, Finland; e-mail: eeva. kormi@hus.fi

Received June 92016

Accepted September 182016

(c) 2017 Published by Elsevier Inc on behalf of the American Association of Oral and Maxillofacial Surgeons

0278-2391/16/30886-2

http://dx.doi.org/10.1016/i.joms.2016.09.026 
is widespread, including trauma surgery. ${ }^{4}$ In facial fracture surgery, few studies have considered the analgesic effect of GCs. However, a study found that the submucosal administration of dexamethasone (DXM) $8 \mathrm{mg}$ after mandibular fractures showed some benefit. ${ }^{5}$ To the best of the authors' knowledge, no studies have analyzed the systemic effect of GCs on pain in facial trauma surgery.

This study evaluated the effect of systemic perioperative DXM on pain after the surgical treatment of blowout fractures. The objective was to observe the effect of DXM on pain assessed using a $10-\mathrm{cm}$ visual analog scale (VAS) during the immediate to 24-hour postoperative period. The authors hypothesized that patients receiving DXM would experience less pain than control patients.

\section{Materials and Methods}

\section{STUDY DESIGN}

The authors designed and implemented a prospective randomized observer-blinded study to analyze the effect of systemic perioperative DXM on pain after treatment for blowout fractures. This study formed part of a larger study evaluating the influence of systemic perioperative DXM on facial fracture healing. The study protocol complied with the Declaration of Helsinki and received approval from the ethics committee of the Department of Surgery and the internal review board of the Division of Musculoskeletal Surgery of Helsinki University Hospital (Helsinki, Finland). All participants signed an informed consent before participation.

\section{STUDY SAMPLE}

The inclusion criterion was a unilateral isolated blowout fracture requiring surgical intervention. Excluded were patients with any of the following characteristics: an allergy to the DXM preparation used (DXM sodium phosphate; Oradexon, N.V. Organon, Netherlands), age younger than 18 years, a steroid-induced psychosis, a gastric ulcer, kidney or liver dysfunction, or pregnant or breast-feeding patients. Also excluded were patients presenting with a previous fracture at the operation site and patients needing analgesics for other reasons.

All patients in this study were recruited from June 1, 2006 through June 30, 2010 from among those presenting at the Department of Oral and Maxillofacial Surgery at Helsinki University Hospital who also satisfied the specified inclusion criterion.

\section{STUDY VARIABLES}

The outcome was pain in the orbital region during the immediate to 24 -hour postoperative period as assessed using the VAS and calculated as the area under the curve (AUC). Perioperative administration of DXM was the primary predictor variable. Other variables included gender, age, preoperative pain (preoperative VAS score), treatment delay, and duration of the operation.

\section{DATA COLLECTION AND MANAGEMENT}

Patients were randomized to receive perioperative DXM or no DXM (controls). Randomization was completed using sealed envelopes and blinded observers.

Participants allocated to the DXM group received DXM $10 \mathrm{mg}$ intravenously during the induction of anesthesia followed by 2 subsequent $10-\mathrm{mg}$ doses intramuscularly at 8 -hour intervals (total, $30 \mathrm{mg}$ ). Controls received no DXM.

General anesthesia was standardized using propofol for induction followed by sevoflurane or desflurane. A local anesthetic (lidocaine or articaine with epinephrine) was injected at the surgical site. Fractures were treated according to typical standards of care. The orbital floor was surgically repaired through a transconjunctival or lower eyelid approach using titanium mesh or an autogenous bone graft from the iliac crest. During anesthesia, pain was controlled using fentanyl. During the induction of anesthesia, acetaminophen $1 \mathrm{~g}$ was administered and continued at 6-hour intervals throughout the hospitalization period. When this provided insufficient analgesia as assessed by a nurse or upon patient request, oxycodone 0.2 to $0.4 \mathrm{mg} / 10 \mathrm{~kg}$ was administered intravenously in the recovery room as the rescue analgesic and oxycodone $1 \mathrm{mg} / 10 \mathrm{~kg}$ was administered intramuscularly on the ward. During hospitalization, as a prophylactic antibiotic, each patient received cefuroxime $1.5 \mathrm{~g} 3$ times per day or, in the case of an allergy, clindamycin $300 \mathrm{mg} 4$ times per day. Patients were hospitalized for at least 24 hours postoperatively. Pain was measured using a $10-\mathrm{cm}$ VAS each time acetaminophen or oxycodone was administered, with the focus on the pain in the orbital region. The pain curve was calculated using the mean VAS score for each minute. If no values were recorded after 24 hours, then the VAS score at 24 hours was set to 0 ; if a VAS score at 24 hours postoperatively was obtained, then the VAS score at 24 hours postoperatively was calculated by interpolation based on the known values before and after the 24-hour time point. For the present analysis, pain was assessed using the mean VAS (centimeters) over time (minutes) as the AUC (VAS AUC) for 24 hours postoperatively.

Data were analyzed using IBM SPSS 23 (IBM Corp, Armonk, NY). After determining the distribution patterns of the continuous variables (Table 1), normally 
Table 1. DISTRIBUTION OF KEY VARIABLES

\begin{tabular}{lcccccc} 
& $\mathrm{n}$ & Min & Max & Mean & SD & $P$ Value \\
\hline Women/men & $8 / 12$ & & & & & \\
Age (yr) & 20 & 22 & 71 & 48.2 & 14.6 & $.193^{*}$ \\
Treatment delay (days) & 20 & 4 & 19 & 7.6 & 3.5 & .001 \\
Preoperative VAS & 20 & 0 & 7.5 & 2.4 & .025 \\
Operation duration (minutes) & 20 & 25 & 83 & 47.2 & 15.9 & $.294^{*}$ \\
VAS AUC for $24 \mathrm{hr}$ & 20 & 386 & 10,388 & 5,110 & 3,173 & $.133^{*}$ \\
\hline
\end{tabular}

Abbreviations: AUC, area under the curve; Max, maximum; Min, minimum; SD, standard deviation; VAS, visual analog scale.

${ }^{*} P>.05$, normally distributed.

Kormi et al. Dexamethasone for Pain in Blowout Fractures. J Oral Maxillofac Surg 2017.

distributed variables (age, VAS AUC, and operation duration) were analyzed using Student $t$ test and the Mann-Whitney $U$ test was used for skewed variables (treatment delay and preoperative VAS). In addition, the $\chi^{2}$ test was used to determine any gender differences. These analyses determined any statistical relevance in the relations between the variables and the randomization group (DXM or no DXM; Table 2) and the outcome (VAS AUC; Tables 3 and 4).

After bivariate analyses, multivariate linear regression analysis was used to determine the association between the independent variables and the outcome. Significance was set at a $P$ value less than .05 .

\section{Results}

The authors identified 27 eligible patients and 24 agreed to participate. Of the 24 patients enrolled, $4 \mathrm{pa}-$ tients were excluded because 3 were administered medications contrary to the study protocol and 1 underwent a reoperation, resulting in 20 patients for the final analysis. Table 1 presents the distribution of key variables used in the analysis. Table 2 presents the results for patients in each randomization group and shows that the groups were comparable except for a shorter operation time in the DXM group (40 vs 56 minutes; $P=.02$ ). In addition, patients in the DXM group reported significantly less pain $(P=.04)$ as assessed by the mean VAS AUC measured 24 hours postoperatively. In the DXM-treated group, pain assessed using the VAS peaked sooner and at a lower level compared with the no-DXM group (Fig 1).

Tables 3 and 4 present other bivariate analyses between the outcome (VAS AUC) and the independent variables. Further analysis (Table 5) showed that DXM remained the only statistically significant factor $(P=.03)$, even when considering other independent variables in multivariate linear regression analyses.

\section{Discussion}

This study evaluated whether systemic perioperative DXM contributed to postoperative pain control after orbital blowout fracture surgery. The present findings showed that DXM for blowout fractures performed well by decreasing the average pain level on the first postoperative day as assessed by the VAS AUC. This finding was statistically significant, representing the only significant factor among the

\section{Table 2. RELATIONS BETWEEN INDEPENDENT VARIABLES AND RANDOMIZATION GROUPS}

\begin{tabular}{|c|c|c|c|c|c|c|c|}
\hline & \multicolumn{3}{|c|}{ DXM } & \multicolumn{3}{|c|}{ No DXM } & \multirow[b]{2}{*}{$P$ Value } \\
\hline & $\mathrm{n}$ & Mean & SD & $\mathrm{n}$ & Mean & SD & \\
\hline Women/men & $5 / 6$ & & & $3 / 6$ & & & .58 \\
\hline Age (yr) & 11 & 46.2 & 12.8 & 9 & 50.7 & 17.0 & .51 \\
\hline Treatment delay (days) & 11 & 6.8 & 2.5 & 9 & 8.4 & 4.5 & .46 \\
\hline Preoperative pain (VAS) & 11 & 2.4 & 2.2 & 9 & 2.4 & 2.5 & .94 \\
\hline Operation duration (minutes) & 11 & 39.8 & 12.6 & 9 & 56.2 & 15.2 & $.02 *$ \\
\hline Pain (VAS AUC) & 11 & 3,792 & 2,795 & 9 & 6,722 & 2,972 & $.04^{*}$ \\
\hline
\end{tabular}

Abbreviations: AUC, area under the curve; DXM, dexamethasone; SD, standard deviation; VAS, visual analog scale.

${ }^{*} P<.05$ (significant). 
Table 3. BIVARIATE ANALYSES BETWEEN GENDER, AN INDEPENDENT VARIABLE, AND PAIN (VAS AUC)

\begin{tabular}{lrccccc} 
& & & \multicolumn{2}{c}{$95 \%$ CI } \\
\cline { 5 - 6 } Gender & $\mathrm{n}$ & Mean (SD) & Difference & Lower Limit & Upper Limit & $P$ Value \\
\hline Women & 8 & $6,056(2,767)$ & & & & \\
Men & 12 & $4,048(3,381)$ & 1,576 & $-1,451$ & 4,603 \\
\hline
\end{tabular}

Abbreviations: AUC, area under the curve; CI, confidence interval; SD, standard deviation; VAS, visual analog scale.

Kormi et al. Dexamethasone for Pain in Blowout Fractures. J Oral Maxillofac Surg 2017.

confounding variables contributing to analgesia $(P=.03)$. In addition, in the DXM group, the average VAS curve peaked sooner and at a lower level.

Specifically, the authors found that the pre-emptive use of DXM effectively controlled postoperative pain. Waldron et $\mathrm{al}^{6}$ evaluated the analgesic impact of a single intravenous dose of DXM in a meta-analysis. In their analysis of 45 randomized controlled studies involving 5,796 patients undergoing various procedures under general anesthesia and receiving DXM 1.25 to $20 \mathrm{mg}$, patients reported less pain and relied less on opioids postoperatively. Their findings parallel the present findings on pain control.

In oral and maxillofacial surgery, most studies considering GCs evaluated the effects related to third molar surgery. However, the literature on pain control remains inconsistent. Surgery causes injury to the tissue in the perioperative site, leading to inflammation. Inflammation is beneficial to the healing process but is also a cause of postoperative discomfort. Acute postoperative pain could be primarily a consequence of this inflammatory reaction. Although GCs are potent anti-inflammatory drugs, they interfere with many signaling pathways. ${ }^{2,7}$ The primary route for action could be suppression of the arachidonic acid cascade by inhibiting the activity of phospholipase $A_{2}$. It also could be the suppression of the cyclo-oxygenase (COX) and lipox-

\begin{tabular}{|c|c|c|c|}
\hline & $\mathrm{n}$ & $\beta$ Estimate & $P$ Value \\
\hline Age & 20 & 0.21 & .930 \\
\hline Treatment delay & 20 & -0.08 & .743 \\
\hline Preoperative pain (VAS) & 20 & 0.59 & $.006^{*}$ \\
\hline Operation time & 20 & 0.46 & $.040^{*}$ \\
\hline
\end{tabular}

Kormi et al. Dexamethasone for Pain in Blowout Fractures. J Oral Maxillofac Surg 2017. ygenase pathways and thus their end products-that is, leukotrienes, thromboxanes, and prostaglandins. Of these, prostaglandins, in particular PGE2 produced through the COX-2 pathway, could carry primary importance for pain. Dionne et $\mathrm{al}^{2}$ found lower prostanoid levels at the third molar extraction site after administration of systemic DXM at doses of $4 \mathrm{mg}$ at 12 and 1 hours preoperatively compared with placebo that had no analgesic effect. The nonsteroidal anti-inflammatory drug ketorolac $30 \mathrm{mg}$ suppressed prostanoid levels, particularly PGE2, more effectively and performed well in pain control, which suggests that an insufficient dose of DXM does not suppress the COX-2 pathway.

In third molar surgical studies, the dose, preparation used, and administration routes differ widely. An analgesic effect was reported in response to a preoperative intravenous 125-mg dose of methylprednisolone and to oral 8- and 6-mg doses of DXM, respectively. ${ }^{8-10}$ Controversial results were found with the local administration of DXM 4 to $10 \mathrm{mg}$ in relation to pain reduction. ${ }^{11}$ Ustün et $\mathrm{al}^{12}$ found no difference in reported pain levels in patients receiving methylprednisolone 1.5 versus $3 \mathrm{mg} / \mathrm{kg}$ intravenously for third molar surgery. In contrast, in the meta-analysis by Waldron et al, ${ }^{6}$ the pain level responded to a dose of DXM, but opioid consumption did not decrease with larger doses of DXM.

In mandibular fracture surgery, the submucosal injection of DXM $8 \mathrm{mg}$ at the surgical incision site decreased pain significantly (VAS score, 2 vs 0 ) at the measurement point of 72 hours, but not at 2, 4, 8, 12,24 , or 48 hours or 7 days postoperatively. ${ }^{5}$ This effect appeared to be mild, which could be explained by the lower DXM dose than administered in the present study and that DXM was administered after an algogenic stimulus. DXM delivered some of its effect with a delay, because it acts in part by downregulating genes that induce inflammation mediator production. Administrating DXM preoperatively could prove more advantageous, as seen in the studies by Skjelbred and Løkken $^{13,14}$ and in the meta-analysis by Waldron et $\mathrm{al}^{6}{ }^{6}$ Also, the systemic administration of GCs seems to be more effective. ${ }^{1}$ 


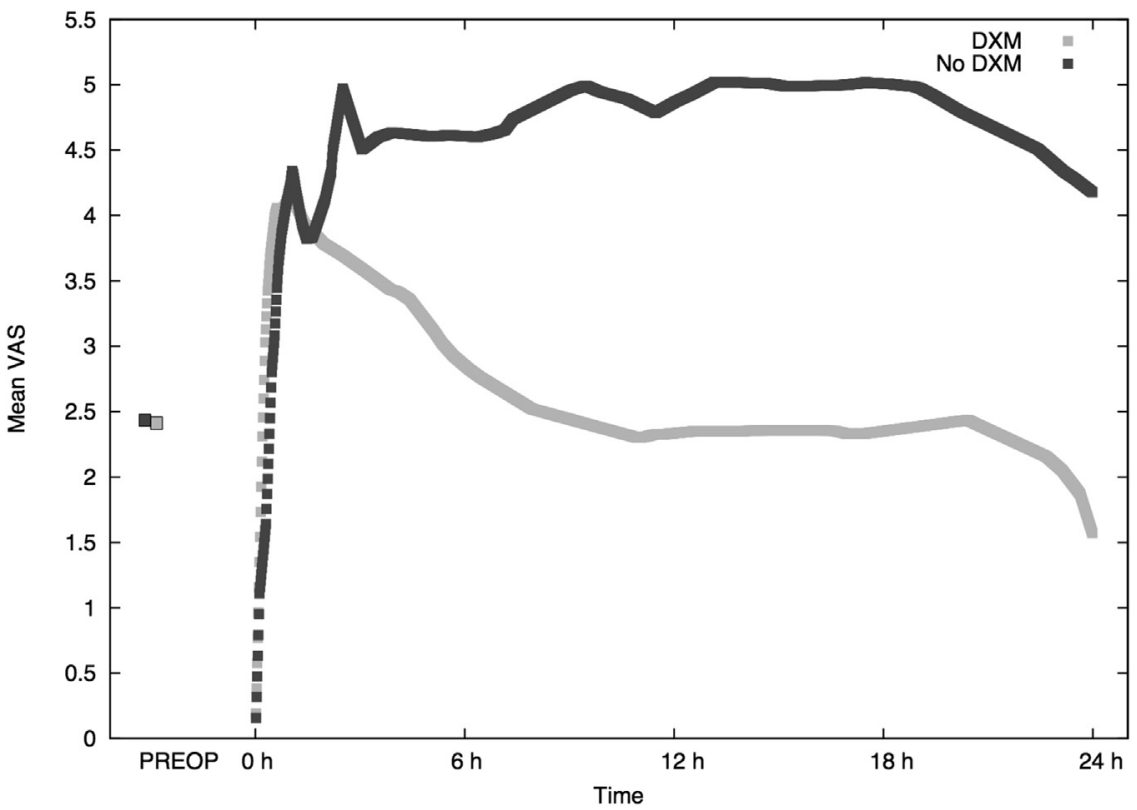

FIGURE 1. Average VAS curve showing less pain in the DMX group. DMX, dexamethasone; VAS, visual analog scale.

Kormi et al. Dexamethasone for Pain in Blowout Fractures. J Oral Maxillofac Surg 2017.

In this study, no complications occurred during the immediate postoperative period in relation to the use of DXM. However, different possible complications of GCs exist and can occur even with short-term use. ${ }^{15-17}$ The risk-to-benefit ratio must be weighed carefully for each patient.

The limitations of this study include its rather small study population and not using a placebo in the study protocol. In addition, the indication for a rescue analgesic was not standardized but was administered depending on the assessment by the nursing staff or upon patient request. In the present study, opioid consumption during the 24-hour period was similar in the 2 groups $(0.15 \mathrm{vs} 0.16 \mathrm{mg} / \mathrm{kg}$ ). However, that indicates that less pain was not achieved with a higher consumption of rescue analgesic. Postoperative pain and oxycodone consumption were generally low; thus, a study considering the effect of DXM in major facial trauma surgery causing more pain would verify the role of DXM in analgesia.

Postoperative pain management represents an important part of surgery. Inadequate pain control

Table 5. MULTIVARIATE LINEAR REGRESSION ANALYSIS PREDICTING PAIN (VAS AUC)

\begin{tabular}{|c|c|c|c|c|c|}
\hline & \multirow[b]{2}{*}{ Coefficient Value } & \multicolumn{2}{|c|}{$95.0 \% \mathrm{CI}$} & \multirow[b]{2}{*}{ SE } & \multirow[b]{2}{*}{$P$ Value } \\
\hline & & Lower Limit & Upper Limit & & \\
\hline (Constant) & $6,182.9$ & -761.7 & $13,127.4$ & $3,214.5$ & .077 \\
\hline Operation time (minutes) & 32.2 & -59.9 & 124.4 & 42.7 & .464 \\
\hline Age (yr) & -19.1 & -115.3 & 77.2 & 44.5 & .676 \\
\hline Gender $(0=$ male, $1=$ female $)$ & $1,556.2$ & $-1,965.4$ & $5,077.8$ & $1,630.1$ & .357 \\
\hline Treatment delay (days) & -260.5 & -657.0 & 135.9 & 183.5 & .179 \\
\hline Preoperative pain (VAS) & 575.9 & -100.3 & $1,252.0$ & 313.0 & .089 \\
\hline $\operatorname{DXM}(0=$ no, $1=$ yes $)$ & $-3,085.4$ & $-5,883.6$ & -287.3 & $1,295.2$ & $.033^{*}$ \\
\hline
\end{tabular}

Note: A predictive model to determine pain during the first 24 hours postoperatively was formulated as: VAS $\mathrm{AUC}=6,182.9+($ operation time $\times 32.2)-($ age $\times 19.1)+($ gender $\times 1,556.2)-($ treatment delay $\times 260.5)+($ preoperative VAS $\times 575.9)-($ DXM $\times 3,085.4)$.

Abbreviations: AUC, area under the curve; CI, confidence interval; DXM, dexamethasone; SE, standard error; VAS, visual analog scale.

${ }^{*} P<.05$ (significant). 
causes morbidity and patient dissatisfaction. Controlling pain effectively and minimizing any possible adverse effects remain important treatment objectives. Sufficient analgesia aids in a faster recovery, an earlier discharge from the hospital, and a lower risk of neuropathic pain. ${ }^{18}$ The present findings show the benefits of DXM as a pre-emptive analgesic in orbital blowout fracture surgery. Future prospective studies with a larger study population, different doses, and accompanying major facial trauma could confirm the present promising results on pain control. Such future studies also could pinpoint the exact dose required to reap the potential benefits and minimize the risks associated with the use of DXM in facial trauma surgery.

\section{References}

1. Alexander RE, Throndson RR: A review of perioperative corticosteroid use in dentoalveolar surgery. Oral Surg Oral Med Oral Pathol Oral Radiol Endod 90:406, 2000

2. Dionne RA, Gordon SM, Rowan J, et al: Dexamethasone suppresses peripheral prostanoid levels without analgesia in a clinical model of acute inflammation. J Oral Maxillofac Surg 61:997, 2003

3. Markiewicz MR, Brady MF, Ding EL, et al: Corticosteroids reduce postoperative morbidity after third molar surgery: A systematic review and meta-analysis. J Oral Maxillofac Surg 66:1881, 2008

4. Assimes T, Lessard ML: The use of perioperative corticosteroids in craniomaxillofacial surgery. Plast Reconstr Surg 103:313, 1999

5. Dongol A, Jaisani MR, Pradhan L, et al: A randomized clinical trial of the effects of submucosal dexamethasone after surgery for mandibular fractures. J Oral Maxillofac Surg 73:1124, 2015
6. Waldron NH, Jones CA, Gan TJ, et al: Impact of perioperative dexamethasone on postoperative analgesia and side-effects: Systematic review and meta-analysis. Br J Anaesth 110:191, 2013

7. Hargreaves KM, Costello A: Glucocorticoids suppress levels of immunoreactive bradykinin in inflamed tissue as evaluated by microdialysis probes. Clin Pharmacol Ther 48:168, 1990

8. Beirne OR, Hollander B: The effect of methylprednisolone on pain, trismus, and swelling after removal of third molars. Oral Surg Oral Med Oral Pathol 61:134, 1986

9. Baxendale BR, Vater M, Lavery KM: Dexamethasone reduces pain and swelling following extraction of third molar teeth. Anaesthesia 48:961, 1993

10. Schmelzeisen R, Frölich JC: Prevention of postoperative swelling and pain by dexamethasone after operative removal of impacted third molar teeth. Eur J Clin Pharmacol 44:275, 1993

11. Graziani F, D'Aiuto F, Arduino PG, et al: Perioperative dexamethasone reduces post-surgical sequelae of wisdom tooth removal. A split-mouth randomized double-masked clinical trial. Int J Oral Maxillofac Surg 35:241, 2006

12. Ustün $Y$, Erdogan $O$, Esen E, et al: Comparison of the effects of 2 doses of methylprednisolone on pain, swelling, and trismus after third molar surgery. Oral Surg Oral Med Oral Pathol Oral Radiol Endod 96:535, 2003

13. Skjelbred P, Løkken P: Post-operative pain and inflammatory reaction reduced by injection of a corticosteroid. A controlled trial in bilateral oral surgery. Eur J Clin Pharmacol 21:391, 1982

14. Skjelbred P, Løkken P: Reduction of pain and swelling by a corticosteroid injected 3 hours after surgery. Eur J Clin Pharmacol 23: 141,1982

15. Spiegel RJ, Vigersky RA, Oliff AI, et al: Adrenal suppression after short-term corticosteroid therapy. Lancet 1:630, 1979

16. Warrington TP, Bostwick JM: Psychiatric adverse effects of corticosteroids. Mayo Clin Proc 81:1361, 2006

17. Snäll J, Kormi E, Koivusalo AM, et al: Effects of perioperatively administered dexamethasone on surgical wound healing in patients undergoing surgery for zygomatic fracture: A prospective study. Oral Surg Oral Med Oral Pathol Oral Radiol 117: 685,2014

18. Ong CK, Seymour RA: Pathogenesis of postoperative oral surgical pain. Anesth Prog 50:5, 2003 